\title{
45. OSTRACODA FROM DSDP LEG 42B
}

\author{
Radu Olteanu, Geological and Geophysical Institut, Bucuresti, Romania
}

\section{INTRODUCTION}

The Pleistocene ostracode assemblage, described in the systematic portion of this report, includes several taxa described previously from different stratigraphical levels of middle Sarmatian, Meotian, Pontian, and Dacian of the circum-Carpathian and from Aktshagylian and Apsheronian of the PontoCaspian regions. In addition, many species have been found in the Recent faunas of the Black Sea and the Caspian Sea.

On the basis of their known ranges, one could be tempted to assume that the ostracode assemblage contains an admixture of older reworked elements. For many reasons, however, such a conclusion cannot be supported under the present state of knowledge. The Ponto-Caspian Basin developed, after becoming isolated from the Mediterranean Sea, into a brackish and fresh-water basin, relict of the Neogene Paratethys; a brief link with the Mediterranean Sea existed only in Meotian time. Later on, in the Black Sea area, successive basins followed: the Pontic Basin with connections to the Caspian and Pannonian basins existed in early Pontian (with the so-called Egeean migration of the fauna) and probably middle Pontian time, succeeded by the Kimmeric, Kuialnik, and Gurian basins (the latter two having again connections with the Caspian Basin), each of which gradually became a fresh-water basin. They, in turn, were followed by the fresh-water Ceaudian and Palaeoeuxin basins.

Mediterranean waters penetrated into the Black Sea waters in Uzumlar time. A new isolation followed and then the link with Mediterranean was re-established in Karangat time (Riss-Würm). During the Würm glacial epoch a break in this connection occurred and the brackish to fresh waters of Neoeuxinic Basin were prevalent. Connection with the Mediterranean Sea was re-established in Holocene time.

Upper Neozoic ostracode faunas of the PontoCaspian Basin were developed by repeated populatings by varying elements from nearby basins; the migrations and populations in the different periods led to the mixed assemblages. Analysis of the components of the fauna permits a separation of ostracode groups relative to their different geographic origins. They originated initially from allopatric speciation within a basin's limits and then migrated into neighboring zones. Thus the distribution in time of different species was not the same in all basins. It must be emphasized that the main part of the Pleistocene ostracode fauna from the PontoCaspian Basin is of Pontian origin. The early Pontian represents the maximum epoch of Pontic basin extension, with the largest faunal migration. Early Pontian faunal elements penetrated from western Europe toward Aral Lake. However, the genera and species from the Ponto-Caspian Basin are not taxa inherited from the Meotian of the same domain but, rather, immigrated from the neighboring Pannonian and Egeean basins (Ebersin et al., 1966). This phenomenon might be explained by premature isolating and freshening of the waters from the Pannonian Basin, relative to the outer Carpathian regions. Once the connections were re-established between the Pannonian and Ponto-Caspian basins, good circulation of fauna followed. With the permanent freshening tendency of the Dacic Basin waters after Pontian time (in the Romanian stage there are only fresh-water faunas), the Pontian type of fauna persisted into the Euxinic and Caspian basins where brackish waters lasted beyond Kimmerian time into Pleistocene and beyond.

The Pannonian faunal migration toward the east explains the presence in the Pleistocene of the PontoCaspian Basin of such species as Amplocypris subacuta and Candona $(C$. $)$ balcanica, as well as specimens of the Candona $(C$.) lobata, Candona $(C$.) labiata, Candona $(C$.) flectimarginata, Candona $(C$.) nonreticulata group.

Callistocythere diffusa (probably a primitive taxon) and Hemicytherura and Cytheroma genera are not found in fossil deposits in Ponto-Caspian regions; they are, however, abundant with numerous species in the Recent fauna of the Black Sea.

Loxoconcha ornata ornata, a prevalent species in our samples, has been described only from middle Sarmatian (Bessarabian) deposits. It survived, unchanged, in isolated ecologic niches up to Pleistocene time.

Paraconadona, relatively frequent in our samples, lives in fresh-water rivers, lakes, pools, etc. In the evolution of the Black Sea its first appearance was in early Quaternary time (Ceaudian and Palaeoeuxinic basins).

Candona species are known from Mesozoic to present time, living in a range of environments. They live mostly in fresh water, but some species live in brackish water; they do not swim but move freely in muddy bottoms.

Bacunella has been found, in several places, in fresh water and brackish deposits of the Pliocene in Europe, and lives today in the Caspian Sea.

Cypria is known from lower Tertiary into Recent deposits. Most species live in fresh-water lakes, pools, etc., but prefer an environment with abundant vegetation.

Leptocythere is typically marine but also is abundant in brackish water, it has a worldwide distribution. 
Fossil species are known from Oligocene time to the present.

Loxoconcha extends from early Tertiary to Recent time. It has a worldwide distribution, inhabiting the littoral zone. zone.

\section{CONCLUSIONS}

Two ostracode assemblages can be distinguished in DSDP Leg 42B samples; a Pleistocene association, comprising mixed ostracode faunas of different paleogeographical origins, and a pre-Recent ostracode community containing the genera Hemicytherura and Cytheroma.

At the moment, in the Pleistocene cores, it is not possible to make any stratigraphical divisions on the basis of the ostracode faunas.

\section{SYSTEMATICS}

\section{Suborder PODOCOPA Sars, 1866}

Family CYPRIDIDAE Baird, 1850

Subfamily CYPRIDINAE Baird, 1850

Genus AMPLOCYPRIS Zalanyi, 1944

Amplocypris aff. subacuta Zalanyi

(Plate 9, Figures 3, 4)

Amplocypris subacuta Zalanyi, 1944, p. 33, pl. 3, fig. 9-12.

Amplocypris cfr. subacuta Zalanyi, Sokac, 1972, p. 35, pl. 11, fig. 14.

Dimensions: $\mathrm{L}=0.78 \mathrm{~mm} ; \mathrm{h}=0.36 \mathrm{~mm}$.

Distribution: This species has so far been found only in the Pannonian deposits (particularly in the sediments of the southeastern part of the Pannonian basin).

\section{Subfamily CANDONINAE Kaufmann, 1960 \\ Genus CANDONA Baird, 1845}

\section{Subgenus CASPIOCYPRIS Mandelstam, 1956}

Candona (Caspiocypris) filona (Livental) (Plate 1, Figure 1)

Caspiocypris filona (Livental), Suzin, 1956, p. 26, pl. 13, fig. 1, 2; Mandelstam et al., 1962, p. 82, pl. 3, fig. 4, 5 .

Dimensions: $\mathrm{L}=0.56 \mathrm{~mm} ; \mathrm{h}=0.39 \mathrm{~mm}$.

Distribution: The Pontian of Kuban, Aktshagylian, and Apsheronian deposits from Turkmenistan. Sokac (1972, p. 41) states that Caspiocypris filona is similar to C. labiata (Zalanyi). (It represents a modification of $C$. labiata due to environmental conditions.)

\section{Candona (Caspiocypris) elongata (Schweyer)}

(Plate 1, Figure 5; Plate 2, Figure 4)

Bythocypris elongata Schweyer, 1948, p. 26, pl. 4, fig. 7, 8.

Candona elongata (Schweyer), Mandelstam et al., 1962, p. 138, pl. 17, fig. 2.

Dimensions: $\mathrm{L}=1.12 \mathrm{~mm} ; \mathrm{h}=0.54 \mathrm{~mm}$.

Distribution: Upper Pliocene and lower Pleistocene of Turkmenistan.

\section{Subgenus CASPIOLLA Mandelstam, 1960}

Candona (Caspiolla) balcanica (Zalanyi)

(Plate 1, Figure 6; Plate 9, Figures 1, 2)

Paracypria balcanica Zalanyi, 1929, p. 52-55, text-fig. 19, 20, pl. 2, fig. 7.

Camtocypria balcanica (Zalanyi), Zalanyi, 1959, p. 225, Gramann, 1969 , p. 492, pl. 33, fig. 1-7.

Caspiolla balcanica (Zalanyi), Mandelstam and Schneider, 1963, p. 90, pl. 7, fig. 11, 12; Stanceva, 1964, p. 209, pl. 1, fig. 1, 2.
Candona (Caspiolla) balcanica (Zalanyi), Sokac, 1972, p. 46, pl. 18, fig. 7-14.

Candona balcanica (Zalanyi), Olteanu, 1974, pl. 1, fig. 4, pl. 2, fig. 4,5 .

Dimensions: $\mathrm{L}=1.37 \mathrm{~mm} ; \mathrm{h}=0.66 \mathrm{~mm}$.

Distribution: This species was described from the upper Pontian of Serbia (Congeria balatonia Zone). It has also been found in the Meotian deposits of the Dacic basin, Pontian deposits in southern parts of USSR (Caucasus, Azerbaijan, Turkmenistan, Basarabia), in Bulgaria, Greece, Romania, and Yugoslavia.

\section{Candona (Caspiolla) confragosa Markova} (Plate 9, Figure 7)

Candona confragosa Markova, Mandelstam et al., 1962, p. 137, pl. 16 , fig. 7,8 .

Dimensions: $\mathrm{L}=1.12 \mathrm{~mm} ; \mathrm{h}=0.67 \mathrm{~mm}$.

Distribution: The upper Pliocene and lower Pleistocene of Turkmenistan.

\section{Candona (Caspiolla) liventalina (Evlachova)}

(Plate 1, Figure 7, Plate 2, Figure 7)

Bythocypris liventalina (Evlachova), Svejer, 1949, p. 26, pl. 4, fig. 1. Caspiolla liventalina (Evlachova), Mandelstam et al., 1962, p. 76, pl. 2, fig. 6.

Dimensions: $\mathrm{L}=0.71 \mathrm{~mm} ; \mathrm{h}=0.39 \mathrm{~mm}$.

Distribution: This species was found at several places in the upper Pliocene and post-Pliocene of the USSR (western Turkmenistan, Azerbaijan).

\section{Candona (Caspiolla) gracilis Livental}

(Plate 1, Figures 8, 9; Plate 2, Figures 1-3)

Candona gracilis Livental, 1929, p. 52, pl. 1, fig. 42, 43.

Caspiolla gracilis (Livental), Mandelstam et al., 1962, p. 77, pl. 2, fig. 2.

Dimensions: $\mathrm{L}=0.81 \mathrm{~mm} ; \mathrm{h}=0.46$ (female right valve); $\mathrm{L}=$ $0.84 \mathrm{~mm} ; \mathrm{h}=0.44 \mathrm{~mm}$ (male right valve).

Distribution: The upper Pliocene and lower Pleistocene deposits of western Turkmenistan and Azerbaijan (USSR).

Subgenus PONTONIELLA Mandelstam, 1960

\section{Candona (Pontoniella) acuminata striata Mandelstam}

$$
\text { (Plate 3, Figures 3-7) }
$$

Pontoniella acuminata var. striata Mandelstam var. $\mathrm{n}$, Mandelstam and Schneider, 1963, p. 86, pl. 6, fig. 4, Gramann, 1969, p. 494, pl. 32 , fig. 1,2 .

Dimensions: $\mathrm{L}=0.78 \mathrm{~mm} ; \mathrm{h}=0.33 \mathrm{~mm}$.

Distribution: This species was found in the Pontian deposits of the USSR (Crimea, Caucasus, Basarabia) and in Bulgaria, Romania, and Greece.

Remarks: $C$. (P.) acuminata striata is nearest to the species $C$. $(P$.) hastata Krstic (described from the upper Pontian of Yugoslavia).

\section{Candona (Pontoniella) schemachensis Mandelstam} (Plate 9, Figure 5)

Pontoniella schemachensis Mandelstam, Mandelstam et al., 1962, p. 72 , pl. 1, fig. 3,4 .

Dimension: $\mathrm{L}=0.56 \mathrm{~mm} ; \mathrm{h}=0.19 \mathrm{~mm}$.

Distribution: The Kuialnikian of Apscheronian of Turkmenistan, cir-Caucasus, and Basarabia (USSR).

Remarks: The striation of the valve is due to certain ecological conditions.

\section{Candona (Pontoniella) sp}

(Plate 9, Figure 6)

Dimensions: $\mathrm{L}=0.44 \mathrm{~mm} ; \mathrm{h}=0.20 \mathrm{~mm}$.

Remarks: This specimen is possibly a juvenile form of the Candona (Pontoniella) schemachensis Mandelstam.

\section{Genus PARACANDONA Hartwig, 1899}

Paracandona aff. verrucosa (Stanceva)

(Plate 3, Figures 1, 2; Plate 8, Figure 7)

Candona verrucosa Stanceva, 1964.

Dimensions: $\mathrm{L}=1.01 \mathrm{~mm} ; \mathrm{h}=0.57 \mathrm{~mm}$. 
Distribution: The Pliocene of Bulgaria. I found this species in the lower Dacian of the Dacic basin (Romania). The genus is found in Pliocene to Recent sediments worldwide.

\section{Genus BACUNELLA Schneider, 1958}

Bacunella dorsoarcuata (Zalanyi)

(Plate 6, Figures 3, 4)

Pontocypris dorsoarcuata Zalanyi, 1929, p. 37-40, fig. 11, 12 .

Bythocypris guriana Svejer, 1949, p. 62, pl. 1, fig. 1-8.

Caspiella dorsoarcuata (Zalanyi) Suzin, 1956, p. 21, pl. 13

fig. $15,16$.

Bacunella dorsoarcuata (Zalanyi), Mandelstam et al., 1962, p. 73, pl. 1, fig. 6, 7; Mandelstam and Schneider, 1963, p. 91, pl. 8, fig. 5, pl. 9, fig. 3, 4; Sejdaeva-Kulieva, 1966, p. 110, pl. 5, fig. 4; Gramann, 1969, p. 495, pl. 32, fig. 5; Sokac, 1972, p. 63 , pl. 29 , fig. $8-12$.

Dimensions: $\mathrm{L}=0.56 \mathrm{~mm} ; \mathrm{h}=0.35 \mathrm{~mm}$.

Distribution: This species was described from the Pontian deposits of Serbia, and afterwards was found in the Pontian from Hungary, Bulgary, Greece, and Romania. It is also know from the Pliocene and post-Pliocene of the southern parts of the USSR (Caucasus, Crimea, Turkmenistan), as well as from the Recent fauna of the Caspian Sea.

\section{Family CYTHERIDAE Baird, 1850}

\section{Subfamily LEPTOCYTHERINAE Hanai, 1957}

\section{Genus LEPTOCYTHERE Sars, 1925}

\section{Leptocythere striatocostata (Svejer)}

(Plate 4, Figure 7)

Cythere stiatocostata Svejer, 1949, p. 27, pl. 9, fig. 8; Schornikov, 1969 , p. 187 , pl. 14, fig. 2

Dimensions: $\mathrm{L}=0.52 \mathrm{~mm} ; \mathrm{h}=0.31 \mathrm{~mm}$.

Distribution: The species is known from the Black Sea and Caspian Sea. It is also found in the Pliocene and post-Pliocene deposits from southern USSR.

Remarks: The species of the subfamily Leptocytherinae described here, are assigned to the genus Leptocythere sensu larger.

Leptocythere bacuana (Livental)

(Plate 5, Figure 1; Plate 9, Figure 8)

Leptocythere bacuana (Livental), Mandelstam et al., 1962; p. 227, pl. 37, fig. 3; Schornikov, 1969 , pl. 15, fig. 3 .

Dimensions: $\mathrm{L}=0.58 \mathrm{~mm} ; \mathrm{h}=0.30 \mathrm{~mm}$.

Distribution: Fossil specimens are known from the Pliocene and lower Pleistocene (Apsheronian) deposits of Turkmenistan and Azerbaijan. It has also been found in Recent fauna of the Black Sea.

Leptocythere bosqueti (Livental)

(Plate 4, Figure 2; Plate 6, Figures 2, 6; Plate 7, Figure 11)

Cythere bosqueti Livental, 1929, p. 24, fig. 27.

Leptocythere bosqueti (Livental), Suzin, 1956, p. 81, pl. 2, fig. 8; Stanceva, 1965, p. 25, pl. 2, fig. 7; Mandelstam et al., 1962, p. 225, pl. 36; Sejdaeva-Kulieva, 1972, p. 68, pl. 31, fig. 14. Dimensions: $\mathrm{L}=0.53 \mathrm{~mm} ; \mathrm{h}=0.26 \mathrm{~mm}$.

Distribution: This species is known from the Pliocene and post. Pliocene of the southern parts of the USSR (north Caucasus, Turkmenistan), in the Pontian of Bulgary and Serbia. It is also found in the Recent fauna of the Caspian Sea.

\section{Leptocythere andrusovi (Livental)}

(Plate 7, Figure 5)

Cythere andrusovi Livental, 1929, p. 16, pl. 1, fig. 6, 7.

Leptocythere andrusovi (Livental), Suzin, 1956, p. 91, 92, pl. 3, fig. 2; Mandelstam et al., 1962, p. 195-196, pl. 30, fig. 13, 14; Sokac, 1972 , p. 69 , pl. 32 , fig. $1,2$.

Dimensions: $\mathrm{L}=0.66 \mathrm{~mm} ; \mathrm{h}=0.34 \mathrm{~mm}$.

Distribution: The Pontian, Aktshagylian, and Apsheronian of the Azerbaijan, Caucasus, and Turkmenistan. It is also known from the Pontian of Bulgaria and Serbia.
Leptocythere multituberculata (Livental)

(Plate 7, Figure 4)

Cythere multituberculata Livental, 1929, p. 14, pl. 1, fig. 36-38; Svejer, 1949, p. 26, pl. 9, fig. 1-3; Suzin, 1956, p. 81, pl. 2, fig. 1; Mandelstam et al., 1962, p. 229, pl. 37, fig. 6; Stanceva, 1972, p. 24-25, pl. 1, fig. 3; Sejdaeva-Kulieva, 1966, p. 90, pl. 3, fig. 10; Gramann, 1969, p. 468, pl. 34, fig. 12; Sokac, 1972, p. 71 , pl. 32 , fig. 14,15 .

Dimensions: $\mathrm{L}=0.44 \mathrm{~mm} ; \mathrm{h}=0.26 \mathrm{~mm}$.

Distribution: Fossil specimens are known from the Pontian and Apsheronian of Azerbaijan, Turkmenistan, and north Caucasus. It has also been found in the Pontian of Bulgaria, Yugoslavia, and Greece and in the Recent fauna from the Caspian Sea.

Leptocythere cymbula (Livental)

(Plate 4, Figure 9; Plate 7, Figure 3)

Cythere cymbula Livental, 1929, p. 21, pl. 1, fig. 25.

Cythere propinqua Livental, 1929, p. 21, pl. 1, fig. 21, 22.

Leptocythere aff. propinqua Livental, Suzin, 1956, p. 119, pl. 5, fig. 21.

Leptocythere propinqua (Livental), Mandelstam et al., 1962, p. 201, pl. 31, fig. 18 Sejdaeva-Kulieva, 1966, p. 92-93, pl. 3, fig. 14.

Leptocythere (Leptocythere) cymbula (Livental), Schornikov, 1969, p. 35 , 36, fig. 3 .

Leptocythere cymbula (Livental), Sokac, 1972, p. 69, pl. 32, fig. 7-9. Dimensions: $\mathrm{L}=0.49 \mathrm{~mm} ; \mathrm{h}=0.24 \mathrm{~mm}$.

Distribution: This species has been found by Schornikov (1966) in Recent faunas from the Black Sea and the Caspian Sea. He compared these forms with the fossil species from Pliocene and post-Pliocene of Turkmenistan, western Caucasus and concluded that the species Leptocythere propinqua (Livental), $L$. accurata Schneider, $L$. aediculata Stepanajtis represent synonyms of the species $L$. cymbula (Livental).

\section{Leptocythere nostrata (Livental)}

(Plate 4, Figure 3)

Leptocythere nostrata (Livental), Mandelstam et al., 1962, p. 200, pl. 31 , fig. $16,17$.

Dimensions: $\mathrm{L}=0.49 \mathrm{~mm} ; \mathrm{h}=0.20 \mathrm{~mm}$.

Distribution: The Aktshagylian and Apsheronian of Turkmenistan and cir-Caucasus.

\section{Leptocythere ergeniensis (Schweyer)}

(Plate 4, Figure 1; Plate 7, Figure 9)

Cythere ergeniensis Schweyer, 1949, p. 34, pl. 8, fig. 4-6.

Leptocythere ergeniensis (Schweyer), Mandelstam et al., 1962, p. 223, pl. 36, fig. 4 .

Dimensions: $\mathrm{L}=0.56 \mathrm{~mm} ; \mathrm{h}=0.29 \mathrm{~mm}$.

Distribution: The upper Pliocene and lower Pleistocene of Turkmenistan and western Caucasus.

\section{Leptocythere aff. caspia (Livental)}

(Plate 4, Figure 4)

Leptocythere caspia (Livental), Mandelstam et al., 1962, p. 232, pl. 37 , fig. 10.

Dimensions: $\mathrm{L}=0.43 \mathrm{~mm} ; \mathrm{h}=0.29 \mathrm{~mm}$.

Distribution: The Aktshagylian and Apsheronian of Turkmenistan and Azerbaijan.

\section{TOCYTHERE Ruggieri, 1953}

Callistocythere cf. diffusa (G.W. Muiller)

(Plate 5, Figure 4; Plate 7, Figure 10)

Cythere diffusa G.W. Müller, 1894, p. 354, pl. 28, fig. 16.

Callistocythere diffusa (G.W. Müller), Caraion, 1967, p. 74 , textfig. 19.

Dimensions: $\mathrm{L}=0.54 \mathrm{~mm} ; \mathrm{h}=0.31 \mathrm{~mm}$.

Distribution: Mediterranean Sea, Black Sea, and Atlantic Ocean.

\section{Subfamily HEMICYTHERINAE Puri, 1953}

Genus HETEROCYTHEREIS Elofson, 1941

Heterocythereis sp 1

(Plate 6, Figure 5)

Dimensions: $\mathrm{L}=0.51 \mathrm{~mm} ; \mathrm{h}=0.34 \mathrm{~mm}$. 
Subfamily CYTHERIDEINAE Sars, 1925

Genus CYTHERISSA Sars, 1928

Cytherissa bogatschovi (Livental)

(Plate 6, Figure 1)

Cytherissa bogatschovi (Livental), Svejer, 1949, p. 37, pl. 8, fig. 2, Mandelstam et al., 1962, p. 190, pl. 30, fig. 1, 2.

Dimensions: $\mathrm{L}=0.35 \mathrm{~mm} ; \mathrm{h}=0.24 \mathrm{~mm}$ (juvenile form).

Distribution: The middle and upper Pliocene of Turkmenistan and Azerbaijan. I found this species in the upper Pontian and Dacian deposits of the Dacic basin (Romania).

Cytherissa bogatschovi plana Klein

(Plate 5, Figure 5; Plate 6, Figure 1)

Cytherissa bogatschovi plana Klein, Mandelstam et al., 1962, p. 190, pl. 30 , fig. 3 .

Dimensions: $\mathrm{L}=0.65 \mathrm{~mm} ; \mathrm{h}=0.37 \mathrm{~mm}$.

Distribution: The lower Pleistocene (Apsheronian) of Turkmenistan and Azerbaijan.

\section{Subfamily CYTHERURINAE G.W. Müller, 1894}

Genus HEMICYTHERURA Elofson, 1941

Hemicytherura sp 1
(Plate 7, Figure 7)
Dimensions: $\mathrm{L}=0.31 \mathrm{~mm} ; \mathrm{h}=0.27 \mathrm{~mm}$ (juvenile form).

Subfamily LOXOCONCHINAE Sars, 1925

Genus LOXOCONCHA Sars, 1866

Loxoconcha eichwaldi Livental

(Plate 5, Figures 6, 7; Plate 7, Figure 2)

Loxoconcha eichwaldi Livental, 1929, p. 34, pl. 1, fig. 42, 43; Suzin, 1956, p. 72, pl. 6, fig. 10, 11; Mandelstam et al., 1962, p. 155, pl. 22 , fig. $2-5$.

Dimensions: $\mathrm{L}=0.70 \mathrm{~mm} ; \mathrm{h}=0.42 \mathrm{~mm}$.

Distribution: The Aktshagylian and Apsheronian of Turkmenistan and western cir-Caucasus.

Loxoconcha petasa (Livental)

(Plate 5, Figure 8; Plate 7, Figure 1)

Loxoconcha petasus Livental, 1929, p. 33, pl. 1, fig. 39-41.

Loxoconcha petasa Livental, Suzin, 1956, p. 67, pl. 6, fig. 4, 5; Mandelstam et al., 1962, p. 164, pl. 25, fig. 4-6.

Dimensions: $\mathrm{L}=0.68 \mathrm{~mm} ; \mathrm{h}=0.34 \mathrm{~mm}$.

Distribution: This species has been found in the Pontian of Yugoslavia, in the Pliocene of Azerbaijan, Turkmenistan, and north Caucasus, in the post-Pliocene of west Turkmenistan, as well as in the Recent fauna of the Caspian Sea.

\section{Loxoconcha schweyeri (Suzin)}

(Plate 8, Figure 6)

Loxoconcha schweyeri Suzin, 1956, p. 70-71, p. 6, fig. 7; Sokac, 1972 , p. 86 , pl. 45 , fig. $8-12$.

Dimensions: $\mathrm{L}=0.67 \mathrm{~mm} ; \mathrm{h}=0.34 \mathrm{~mm}$.

Distributions: This species was first described from Pontian sediments of northern Caucasus, and then it has been found in the Pontian of Bulgaria and Yugoslavia.

\section{Loxoconcha ornata ornata Schneider}

(Plate 7, Figure 8; Plate 8, Figures 1-5)

Loxoconcha ornata Schneider, 1949, p. 140, pl. 8, fig. 1.

Loxoconcha ornata ornata Schneider, Stanceva, 1972, p. 106, pl. 3?, fig. 11.

Dimensions: $\mathrm{L}=0.44 \mathrm{~mm} ; \mathrm{h}=0.25 \mathrm{~mm}$ (Plate 7, Figures 3-5);

$\mathrm{L}=0.54 \mathrm{~mm} ; \mathrm{h}=0.32 \mathrm{~mm}$ (Plate 7, Figures 1, 2).

Distribution: The middle Sarmatian (sensu Barbot de Marny 1966), and the Besarabian of Bulgaria and southern USSR.

\section{INCERTAE FORMS}

Candona (Caspiolla) ex gr. lobata (Zalanyi)

(Plate 1, Figure 4)

Paracypris lobata Zalanyi, 1929, p. 55-57, text-fig. 21, 22, pl. 2, fig. 8.

Caspiolla lobata (Zalanyi), Mandelstam and Schneider, 1963, p. 89, 90 , p. 8 , fig. 10.

Dimensions: $\mathrm{L}=0.77 \mathrm{~mm} ; \mathrm{h}=0.35 \mathrm{~mm}$.

Distribution: This species was first found in the upper Pontian of Serbia (Congeria balatanica Zone). It has also been found at numerous places in the Pontian deposits of Azerbaijan, Turkmenistan, Crimea, Caucasus, Basarabia (USSR), and in Bulgaria.

Candona (Caspiocypris) ex gr. labiata (Zalanyi)

(Plate 1, Figure 4)

Paracypris labiata Zalanyi, 1929 , p. $48-51$, text-fig. 17,18 , p. 12 , fig. 9.

Caspiocypris labiata (Zalanyi), Mandelstam and Schneider, 1963, p. 98, pl. 10 , fig. 6,7 .

Candona (Caspiocypris) labiata (Zalanyi), Sokac, 1972, p. 40, pl. 15, fig. 8-13.

Dimensions: $\mathrm{L}=0.74 \mathrm{~mm} ; \mathrm{h}=0.33 \mathrm{~mm}$.

Distribution: The species was described from the upper Pontian of Serbia. It has also been found in the Pontian of the Caucasus and Basarabia (USSR).

Candona (Caspiolla) ex gr. flectimarginata Sokac

(Plate 2, Figure 6; Plate 6, Figure 2)

Candona (Caspiolla) flectimarginata Sokac, Sokac, 1972, p. 47, pl. 20, fig. 1-6.

Dimensions: $\mathrm{L}=0.81 \mathrm{~mm} ; \mathrm{h}=0.36 \mathrm{~mm}$.

Distribution: This species was described from the upper Pontian of Yugoslavia. It is also characteristic of these deposits in the south part of the Pannonian basin.

\section{Candona (Lineocypris) ex gr. nonreticulata Sokac} (Plate 1, Figure 2)

Candona (Lineocypris) nonreticulata Sokac, 1972, p. 54, pl. 24, fig. 1-7.

Dimensions: $\mathrm{L}=0.79 \mathrm{~mm} ; \mathrm{h}=0.41 \mathrm{~mm}$ (juvenile form).

Distribution: This species was described from the upper Pannonian.

$$
\begin{gathered}
\text { Cytheroma (?) sp. } \\
\text { (Plate 7, Figure 6) } \\
\text { Dimensions: } \mathrm{L}=0.46 \mathrm{~mm} ; \mathrm{h}=0.21 \mathrm{~mm} .
\end{gathered}
$$

\section{ACKNOWLEDGMENTS}

All specimens described here are located in the Geological and Geophysical Institut (Micropalaeontological Collection), București. All drawings were done by the author.

\section{REFERENCES}

Caraion, E.F., 1967. Fauna R.S.R., Crustacea (Ostracoda), Fam. Cytheridae (ostracode marine şi salmastricole): v. IV, fasc. 10, Ed. Acad. RSR.

Ebersin, A.G., Motas, I.C., Macarovivi, N., and Marinescu, F., 1966. Afinitati panonice si euxinice ale Neogenului superior din Băzinul Dacic: St. Cerc. Geol. Geof. Geogr., s. Geol., v. 11, p. 2.

Gramann, F., 1969. Das Neogen im Strimon-Becken (Griechish-Ostmazedonien): Geol. Jb., v. 87.

Livental, V.E., 1929. Ostracoda Akciagilscogo i Apşeronscogo Iarusov: Iz. Iz. Azerb. Polit. Inst., Bacu.

Mandelstam, M.I., Markova, L.P., Rozyeva, T.R., and Stepanajtys, N.E., 1962. Ostracody pliocenovyh i postpliocenovyh otlojenij Turkmenistana: Izd. Akad. Nauk Turkmeniskoj SSR, Ashabad. 
Mandelstam, M.I. and Schneider, G.F., 1963. Iskopaemye ostracody SSSR. Semejstvo Cyprididae: Trudy VNIGRI, Leningrad.

Sokac, A., 1972. Pannonian and Pontian Ostracode fauna of Mt. Medvenice: Izdav. Zav. Jugoslavenske Acad., Zagreb.

Stanceva, M., 1964. Ostracoda from the Neogene in NorthWestern Bulgaria: Trud. Geol. Blg., ser. Pal., Sofia. 1972. Sarmatian Ostracodes from North-Eastern Bulgaria: Bull. Geol. Inst., ser. Pal., 21, Sofia.

Suzin, A.V., 1956. Ostracodny treticnyh otlozenij Severnogo Predkavkazia: Gost. ord. Trud. k. z. neft. Gostoptehizdat, Moskva.

Sejdaeva-Kulieva, H.M., 1966. Ostracody ponticescogo jarusa Vostocnogo Azerbajzana: Iz. Acad. Nauk Azerb. SSR, Bacu.
Schneider, G.F., 1949. Miotenovaia fauna ostracod Kavkaza i Krima: Tr. VNIGRI, n. ser., v. 34, Leningrad-Moskva.

Schornikov, E.I., 1966. Leptocythere (Crustacea, Ostracoda) Ahovo-Cernomorskogo basejna: Zool. zurn., 45/1, Moskva.

1969. Podclass Ostracoda, ili rakuşcovie rakiOstracoda Latreille, 1816. in Opredelitel faunu Cernogo i Azovskogo Morei, T. II: Acad. Nauk USSR, Inst. Biol. Iujnih Morei, Kiev.

Svejer, A.V., 1949. Osnovi morfologii i sistematiki pliocenovih i postpliocenovih Ostracod. Trudy VNIGRI, n. ser. 30, Gostoptehizdat, Leningrad-Moskva.

Zalanyi, B., 1929. Morpho-systematische Studien uber fossile Muschelkrebse: Geol. Hung., ser. pal., 5, Budapest.

1959. Oberpononische Ostracoden aus Tihany: Mag. All. Fold. Intez. Evk. 1/48, Budapest.

APPENDIX

Occurrences of Ostracode Species in Leg 42B Cores

\begin{tabular}{|c|c|c|c|}
\hline $\begin{array}{c}\text { Sample } \\
\text { (Interval in } \mathrm{cm} \text { ) }\end{array}$ & Species & $\begin{array}{l}\text { Number } \\
\text { of Forms }\end{array}$ & Adult/Juvenile \\
\hline \multicolumn{4}{|l|}{ Hole 379A } \\
\hline \multirow[t]{2}{*}{$8-5,64-66$} & $\begin{array}{l}\text { Heterocythereis } \mathrm{sp}_{1} \\
\text { Cytherissa bogatschovi }\end{array}$ & 1 & Juvenile \\
\hline & var. plana & 1 & Juvenile \\
\hline \multirow{4}{*}{$9-1,120-122$} & Leptocythere bacuana & 1 & Juvenile \\
\hline & Leptocythere cymbula & 1 & Juvenile \\
\hline & Leptocythere caspia & 1 & Juvenile \\
\hline & Callistocythere aff diffusa & 1 & Adult \\
\hline \multirow[t]{3}{*}{$11-5,15-17$} & Leptocythere bacuana & 1 & Juvenile \\
\hline & Loxoconcha eichwaldi & 1 & Juvenile \\
\hline & Candona (Caspiolla) gracilis & 2 & Juvenile \\
\hline \multirow{7}{*}{$11-5,26-27$} & Candona (C.) gracilis & 2 & Adult \\
\hline & Leptocythere multituberculata & $1+1$ & Adult, juvenile \\
\hline & Leptocythere caspia & 1 & Adult \\
\hline & Leptocythere bacuana & 1 & Juvenile \\
\hline & Candona (Caspiocy pris) filona & 1 & Adult \\
\hline & Cytheroma $\mathrm{sp}_{1}$ & 1 & Adult \\
\hline & Cytherissa bogatschovi & 1 & Juvenile \\
\hline $15-2,48-50$ & Unidentified fragments of Candona genus & & \\
\hline $20-3,104-106$ & Candona (C.) aff gracilis & Fragments & \\
\hline $21-2,98-100$ & Candona (C.) aff gracilis & Fragments & \\
\hline \multirow{3}{*}{$36, \mathrm{CC}$} & Loxoconcha ornata ornata & 2 & Juvenile \\
\hline & Leptocythere caspia & 1 & Adult \\
\hline & Leptocythere bacuana & 1 & Adult \\
\hline $37, \mathrm{CC}$ & Bacunella dorsoarcuata & 2 & Juvenile \\
\hline \multirow[t]{2}{*}{$38, \mathrm{CC}$} & Loxoconcha aff eichwaldi & 1 (fragm.) & Adult \\
\hline & Leptocythere bacuana & 1 & Juvenile \\
\hline \multirow[t]{3}{*}{$42-1,102-104$} & Candona $\mathrm{sp}_{3}$ ex gr. nonreticulata & 1 & Adult \\
\hline & Candona (C.) liventalina & 3 & Juvenile \\
\hline & Candona sp $_{2}$ ex gr. labiata & 1 & Adult (?) \\
\hline \multirow[t]{2}{*}{$45, \mathrm{CC}$} & Leptocythere bacuana & 1 & Adult \\
\hline & Candona (C.) liventalina & 1 & Adult \\
\hline \multirow[t]{2}{*}{$46, \mathrm{CC}$} & Loxoconcha eichwaldi & 1 & Adult \\
\hline & Paracandona aff verrucosa & 1 & Adult \\
\hline $48-4,66-68$ & Candona (C.) liventalina & 1 (fragm.) & Adult \\
\hline \multirow[t]{2}{*}{$49-1,56-58$} & Loxoconcha aff eichwaldi & 1 (fragm.) & Adult \\
\hline & Candona (C.) liventalina & 1 (fragm.) & Adult \\
\hline \multirow[t]{2}{*}{$51, \mathrm{CC}$} & Leptocythere bacuana & 1 & Juvenile \\
\hline & Loxoconcha aff eichwaldi & 1 (fragm.) & Adult \\
\hline \multirow[t]{4}{*}{$52, \mathrm{CC}$} & Loxoconcha ornata ornata & 2 & Juvenile \\
\hline & Paracandona aff verrucosa & 1 (fragm.) & Adult \\
\hline & Leptocythere bacuana & 1 & Juvenile \\
\hline & Loxoconcha aff eichwaldi & 1 (fragm.) & Adult \\
\hline \multirow{3}{*}{$53-1,92-94$} & Bacunella dorsoarcuata & 2 & Adult \\
\hline & Leptocythere cymbula & 1 & Juvenile \\
\hline & Candona (C.) liventalina & 2 & Adult \\
\hline \multirow[t]{2}{*}{$54, \mathrm{CC}$} & Loxoconcha aff petasa & 3 (fragm.) & Adult, juvenile \\
\hline & Candona (C.) liventalini & 2 (+numerous fragm.) & Adult \\
\hline
\end{tabular}


APPENDIX - Continued

\begin{tabular}{|c|c|c|c|}
\hline $\begin{array}{c}\text { Sample } \\
\text { (Interval in } \mathrm{cm} \text { ) }\end{array}$ & Species & $\begin{array}{l}\text { Number } \\
\text { of Forms }\end{array}$ & Adult/Juvenile \\
\hline \multicolumn{4}{|c|}{ Hole 379A - Continued } \\
\hline \multirow[t]{4}{*}{$59-1,105-107$} & Candona (C.) liventalina & 1 & Adult \\
\hline & Candona $\mathrm{sp}_{1}$ ex gr. lobata & 1 & Adult \\
\hline & Loxoconcha eichwaldi & 2 & Juvenile \\
\hline & Bacunella dorsoarcuata & 2 & Juvenile \\
\hline \multirow[t]{2}{*}{$59-3,83-85$} & Candona (C.) gracilis & 1 & Adult \\
\hline & Candona $\mathrm{sp}_{3}$ cfr flectimarginata & 2 & Juvenile \\
\hline \multirow[t]{6}{*}{$59, \mathrm{CC}$} & Candona (C.) gracilis & 2 & Juvenile \\
\hline & Leptocythere bacuana & 1 & Juvenile \\
\hline & Leptocythere ergeniensis & 2 & Adult \\
\hline & Leptocythere caspia & 1 & Juvenile \\
\hline & Loxoconcha ornata ornata & 3 & Juvenile \\
\hline & Loxoconcha eichwaldi & 1 & Juvenile \\
\hline \multirow[t]{3}{*}{$60-4,36-38$} & Heterocythereis $\mathrm{sp}_{1}$ & 1 & Juvenile \\
\hline & Candona (C.) gracilis & 2 & Adult \\
\hline & Bacunella dorsoarcuata & 1 & Adult \\
\hline \multirow[t]{4}{*}{$65-4,84-86$} & Leptocythere bacuana & 1 & Adult \\
\hline & Candona (C.) liventalina & 2 & Adult \\
\hline & Bacunella dorsoarcuata & $2(+3$ fragm.) & Adult \\
\hline & Loxoconcha ornata ornata & 1 & Juvenile \\
\hline \multicolumn{4}{|l|}{ Hole 379B } \\
\hline \multirow{2}{*}{$4, \mathrm{CC}$} & Leptocythere aff cymbu & 1 & Juvenile \\
\hline & Candona (C.) gracilis & 3 & Juvenile \\
\hline \multirow[t]{3}{*}{$6, \mathrm{CC}$} & Cyprideis $\mathrm{sp}_{1}$ & 1 & Juvenile \\
\hline & Loxoconcha petasa & 1 & Adult \\
\hline & Candona (C.) gracilis & 1 & Juvenile \\
\hline \multicolumn{4}{|l|}{ Hole 380} \\
\hline \multirow[t]{3}{*}{$1, \mathrm{CC}$} & Heterocythereis $\mathrm{sp}_{1}$ & 11 & Adult (2), juvenile \\
\hline & Leptocythere ergeniensis & 2 & Adult \\
\hline & Leptocythere bosqueti & 2 & Adult \\
\hline $4-2,92-94$ & Heterocythereis $\mathrm{sp}_{1}$ & 1 & Adult \\
\hline $4-3,52-54$ & Heterocythereis $\mathrm{sp}_{1}$ & 3 & Juvenile \\
\hline $4-4,41-43$ & Heterocythereis $\mathrm{sp}_{1}$ & 1 & Juvenile \\
\hline \multirow[t]{9}{*}{$5-1,103-105$} & Leptocythere andrusovi & 1 & Adult \\
\hline & Leptocythere bosqueti & 1 & Adult \\
\hline & Loxoconcha eichwaldi & 2 & Adult \\
\hline & Heterocythereis $\mathrm{sp}_{1}$ & 1 & Juvenile \\
\hline & Leptocythere cymbula & 2 & Juvenile \\
\hline & Leptocythere caspia & 1 & Adult \\
\hline & Leptocythere nostrata & 1 & Adult \\
\hline & Bacunella dorsoarcuata & 2 & Juvenile \\
\hline & Candona (C.) gracilis & 4 & Adult (1), juvenile \\
\hline \multirow[t]{3}{*}{$6-1,133-135$} & Candona elongata & 2 & Adult \\
\hline & Loxoconcha petasa & 2 & Adult + juvenile \\
\hline & Loxoconcha eichwaldi & 2 & Juvenile \\
\hline \multirow[t]{4}{*}{$8-1,87-89$} & Loxoconcha eichwaldi & 2 & Juvenile \\
\hline & Hemicytherura $\mathrm{sp}_{1}$ & 1 & Juvenile \\
\hline & Leptocythere multituberculata & 1 & Adult \\
\hline & Leptocythere cymbula & 3 & Juvenile \\
\hline $35-4,58-60$ & Loxoconcha eichwaldi & 2 & Juvenile \\
\hline $36-3,82-84$ & Loxoconcha schweyeri & 4 & Adult (1), juvenile \\
\hline $37-2,46-48$ & numerous fragments of Candona genus & & \\
\hline \multirow[t]{2}{*}{$40, \mathrm{CC}$} & Bacunella dorsoarcuata & 2 & Adult \\
\hline & Loxoconcha eichwaldi & 3 & Adult (2), juvenile \\
\hline \multicolumn{4}{|l|}{ Hole $380 \mathrm{~A}$} \\
\hline $3, \mathrm{CC}$ & Loxoconcha ornata ornata & 3 & Juvenile \\
\hline & Leptocythere cymbula & 1 & Juvenile \\
\hline $6-3,88-90$ & Loxoconcha eichwaldi & 2 & Juvenile \\
\hline & Loxoconcha ornata ornata & 2 & Juvenile \\
\hline $9, \mathrm{CC}$ & Loxoconcha ornata ornata & 2 & Juvenile \\
\hline & Cytherissa bogatschovi plana & 1 & Adult \\
\hline
\end{tabular}




\begin{tabular}{|c|c|c|c|}
\hline $\begin{array}{c}\text { Sample } \\
\text { (Interval in } \mathrm{cm} \text { ) }\end{array}$ & Species & $\begin{array}{l}\text { Number } \\
\text { of Forms }\end{array}$ & Adult/Juvenile \\
\hline \multicolumn{4}{|c|}{ Hole $380 \mathrm{~A}$ - Continued } \\
\hline \multirow[t]{3}{*}{$11-2,38-40$} & Leptocythere caspia & 2 & Adult \\
\hline & Loxoconcha ornata ornata & 6 & Adult (4), juvenile \\
\hline & Candona (C.) liventalini & 3 & Adult \\
\hline $13, \mathrm{CC}$ & Unidentified fragments of Candona genus & & \\
\hline \multirow{2}{*}{$14-2,99-101$} & Candona (C.) gracilis & 2 & Juvenile \\
\hline & Candona sp ex gr. labiata & 1 & Adult \\
\hline $14-6,85-87$ & Candona (C.) gracilis & 2 & Juvenile \\
\hline $14, \mathrm{CC}$ & Candona (C.) gracilis & 3 & Juvenile \\
\hline $19, \mathrm{CC}$ & Candona (C.) gracilis & 2 & Juvenile \\
\hline $15, \mathrm{CC}$ & Candona (C.) aff labiata & 2 & Juvenile \\
\hline $23-3$ & Unidentified fragments & & \\
\hline $23-4,66-68$ & $\begin{array}{l}\text { Candona (C.) sp. ex gr. lobata } \\
\text { Candona (C) balcanica }\end{array}$ & $\begin{array}{l}6 \\
2\end{array}$ & $\begin{array}{l}\text { Adult (+numerous fragm.) } \\
\text { Adult }\end{array}$ \\
\hline $23, \mathrm{CC}$ & $\begin{array}{l}\text { Candona }(C \text { ) balcanica } \\
\text { Unidentified fragments }\end{array}$ & 2 & Adult \\
\hline $26, \mathrm{CC}$ & Unidentified fragments & & \\
\hline \multirow[t]{2}{*}{$28, \mathrm{CC}$} & Candona (C.) balcanica & 4 & Adult \\
\hline & Candona (C) sp. ex gr. lobata & 3 & Adult \\
\hline \multirow[t]{2}{*}{$32, \mathrm{CC}$} & Candona (C.) sp. ex gr. labiata & 1 & Adult \\
\hline & Loxoconcha ornata ornata & 2 & Juvenile \\
\hline $31, \mathrm{CC}$ & Unidentified fragments & & \\
\hline $60, \mathrm{CC}$ & Unidentified fragments & & \\
\hline \multicolumn{4}{|l|}{ Site 381} \\
\hline \multirow[t]{2}{*}{$1-1,62-64$} & Loxoconcha schweyeri & 1 & Adult \\
\hline & Loxoconcha eichwaldi & 1 & Juvenile \\
\hline \multirow[t]{3}{*}{$2-1,72-74$} & Heterocythereis sp & 2 & Juvenile \\
\hline & Candona (c.) balcanica & 1 & Juvenile \\
\hline & Candona (C.) gracilis & 3 & Juvenile \\
\hline \multirow[t]{3}{*}{$2, \mathrm{CC}$} & Leptocythere cymbula & 2 & Juvenile \\
\hline & Candona (C.) gracilis & 4 & Juvenile \\
\hline & Candona elongate & 2 & Juvenile \\
\hline \multirow[t]{4}{*}{$3, \mathrm{CC}$} & Candona (C.) balcanica & 2 & Adult \\
\hline & Candona (C.) gracilis & 4 & Juvenile \\
\hline & Candona elongata & 1 & Juvenile \\
\hline & Leptocythere cymbula & 1 & Adult \\
\hline $4-2,82-84$ & Leptocythere ergeniensis & 1 & Adult \\
\hline \multirow[t]{4}{*}{$4, \mathrm{CC}$} & Candona (C.) gracilis & 3 & Adult \\
\hline & Candona elongata & 4 & Adult (1), juvenile \\
\hline & Cytherissa bogat schovi & 1 & Juvenile \\
\hline & Leptocythere cymbula & 2 & Adult \\
\hline $5-2,82-84$ & Candona (C.) gracilis & 3 & Juvenile \\
\hline \multirow[t]{4}{*}{$5-4,72-74$} & Candona (C.) liventalini & 3 & Adult \\
\hline & Candona (C.) balcanica & 3 & Juvenile \\
\hline & Candona (Pontoniella) cfr schemachensis & 1 & Adult \\
\hline & Candona (C.) gracilis & 2 & Adult \\
\hline \multirow[t]{2}{*}{$5, \mathrm{CC}$} & Leptocythere multituberculata & 1 & Juvenile \\
\hline & Candona (C.) gracilis & 2 & Juvenile \\
\hline \multirow[t]{3}{*}{$6-2,72-74$} & Bacunella dorsoarcuata & 3 & Juvenile \\
\hline & Loxoconcha ornata ornata & 1 & Juvenile \\
\hline & Candona (C.) liventalina & 1 & Adult \\
\hline $6, \mathrm{CC}$ & Loxoconcha eichwaldi & 1 & Juvenile \\
\hline & Candona (C.) gracilis & 3 & Adult (1), juvenile \\
\hline & Loxoconcha ornata ornata & 2 & Juvenile \\
\hline & Candona (C.) liventalina & 1 & Adult \\
\hline & Candona (C.) balcanica & 1 & Adult \\
\hline & Baounella dorsoarcuata & 1 & Juvenile \\
\hline $7-3,72-74$ & Leptocythere multituberculata & 1 & Juvenile \\
\hline & Leptocythere ergeniensis & 1 & Juvenile \\
\hline & Candona (C.) gracilis & 2 & Juvenile \\
\hline & Candona (Caspiocypris) filona & 1 & Adult \\
\hline & Bacunella dorsoarcuata & 1 & Adult \\
\hline $8-1,72-74$ & Paracandona aff verrucosa & 1 & Adult \\
\hline & Candona (C.) liventalina & 4 & Adult (2), juvenile \\
\hline & Candona (Caspiocypris) filona & 2 & Juvenile \\
\hline
\end{tabular}




\begin{tabular}{|c|c|c|c|}
\hline $\begin{array}{c}\text { Sample } \\
\text { (Interval in } \mathrm{cm} \text { ) }\end{array}$ & Species & $\begin{array}{l}\text { Number } \\
\text { of Forms }\end{array}$ & Adult/Juvenile \\
\hline \multicolumn{4}{|c|}{ Site 381 - Continued } \\
\hline & Candona (Pontoniella) $\mathrm{sp}_{1}$ & 1 & Juvenile \\
\hline & Bacunella dorsoarcuata & 4 & Adult (1), juvenile \\
\hline & Loxoconcha ornata ornata & 3 & Juvenile \\
\hline & Leptocythere striatocostata & 1 & Adult \\
\hline \multirow[t]{2}{*}{$8-3,72-74$} & Candona (C.) liventalina & 3 & Adult \\
\hline & Bacunella dorsoarcuata & 3 & Adult \\
\hline \multirow[t]{5}{*}{$8-5,82-84$} & Candona (C.) liventalina & 4 & Adult \\
\hline & Candona (C.) gracilis & 3 & Juvenile \\
\hline & Candona (Caspiocypris) filona & 2 & Juvenile \\
\hline & Amplocypris subacuta & 2 & Adult (?) \\
\hline & Candona confragosa & 1 & Adult \\
\hline \multirow[t]{12}{*}{$9-1,94-96$} & Candona (C.) liventalina & 4 & Adult \\
\hline & Candona (C.) balcanica & 2 & Adult \\
\hline & Candona (Pontoniella) $\mathrm{sp}_{1}$ & 2 & Adult \\
\hline & Leptocythere ergeniensis & 3 & Adult \\
\hline & Leptocythere andrusovi & 2 & Adult \\
\hline & Leptocythere striatocostata & 2 & Adult \\
\hline & Leptocythere bacuana & 1 & Juvenile \\
\hline & Leptocythere caspia & 1 & Adult \\
\hline & Bacunella dorsoarcuata & 2 & Juvenile \\
\hline & Heterocythereis $\mathrm{sp}_{1}$ & 2 & Juvenile \\
\hline & Loxoconcha eichwaldi & 4 & Juvenile \\
\hline & Loxoconcha petasa & 1 & Adult \\
\hline \multirow[t]{4}{*}{$9-5,77-79$} & Candona (C.) liventalina & 4 & Adult \\
\hline & Candona (C.) gracilis & 1 & Juvenile \\
\hline & Leptocythere bacuana & 1 & Juvenile \\
\hline & Loxoconcha ornata ornata & 2 & Juvenile \\
\hline $10, \mathrm{CC}$ & Loxoconcha ornata ornata & 1 & Juvenile \\
\hline \multirow[t]{4}{*}{$13, \mathrm{CC}$} & Candona (C.) liventalina & 5 & Adult \\
\hline & Candona (C) gracilis & 8 & Juvenile \\
\hline & Bacunella dorsoarcuata & 3 & Juvenile \\
\hline & Candona sp. ex gr. flectimarginata & 1 & Adult \\
\hline \multirow{2}{*}{$14, \mathrm{CC}$} & Candona $(C$.$) gracilis$ & 1 & Juvenile \\
\hline & Candona (C.) liventalina & 3 & Juvenile \\
\hline \multirow[t]{2}{*}{$15-6,92-94$} & Candona (C.) liventalini & 1 & Juvenile \\
\hline & Candona $\mathrm{sp}_{1}$ cf. lobata & 1 & Adult (?) \\
\hline \multirow[t]{7}{*}{$15, \mathrm{CC}$} & Candona $\mathrm{sp}_{3}$ & 1 & Adult \\
\hline & Candona (C.) liventalina & 3 & Adult \\
\hline & Candona (C.) gracilis & 7 & Juvenile \\
\hline & Candona (P.) acuminata striata & 2 & Adult \\
\hline & Candona (C.) balcanica & 2 & Adult \\
\hline & Paracandona aff verrucosa & 1 & Adult \\
\hline & Bacunella dorsoarcuata & 7 & Juvenile \\
\hline \multirow[t]{3}{*}{$16-4,72-74$} & Candona (C.) liventalina & 3 & Juvenile \\
\hline & Candona (C.) gracilis & 3 & Juvenile \\
\hline & Bacunella dorsoarcuata & 3 & Juvenile \\
\hline \multirow[t]{2}{*}{$16-5,74-76$} & Candona (C.) liventalina & 2 & Adult \\
\hline & Loxoconcha ornata ornata & 1 & Juvenile \\
\hline \multirow[t]{6}{*}{$17-3,72-74$} & Candona (P.) schemachensis & 1 & Adult \\
\hline & Candona (P.) acuminata striata & 4 & Adult \\
\hline & Candona $\mathrm{sp}_{2} \mathrm{cf}$. labiata & 1 & Adult \\
\hline & Leptocythere bosqueti & 3 & Adult \\
\hline & Loxoconcha petasa & 1 & Adult \\
\hline & Loxoconcha eichwaldi & 2 & Adult \\
\hline \multirow[t]{5}{*}{$17, \mathrm{CC}$} & Candona (P.) acuminata striata & 2 & Adult \\
\hline & Loxoconcha ornata ornata & 1 & Adult \\
\hline & Loxoconcha petasa & 3 & Adult (2), juvenile \\
\hline & Bacunella dorsoarcuata & 2 & Adult \\
\hline & Heterocythereis $\mathrm{sp}_{1}$ & 1 & Juvenile \\
\hline $18-2,52-54$ & Candona (C.) balcanica & 2 & Adult \\
\hline $18-5,72-74$ & Candona $\mathrm{sp}_{2}$ cf. labiata & 1 & Adult \\
\hline $18, \mathrm{CC}$ & Candona $\mathrm{sp}_{2} \mathrm{cf}$. labiata & 3 & Adult \\
\hline & Candona (C.) liventalina & 1 & Juvenile \\
\hline $19-1,72-74$ & Candona (C.) liventalina & 1 & Adult \\
\hline & Bacunella dorsoarcuata & 1 & Adult \\
\hline $39, \mathrm{CC}$ & Unidentified fragments & & \\
\hline
\end{tabular}




\section{PLATE 1}

Figure $1 \quad$ Candona (Caspiocypris) filona Livental-exterior lateral view of right valve.

Figure 2 Candona $\mathrm{sp}_{3}$ ex gr. nonreticulata Sokac-exterior lateral view of left juvenile valve.

Figure 3 Candona $\mathrm{sp}_{2}$ ex gr. labiata (Zalanyi)-exterior lateral view of right valve.

Figure 4 Candona $\mathrm{sp}_{3}$ ex gr. lobata (Zalanyi)-exterior lateral view of right valve.

Figure 5 Candona (Caspiocypris) elongata (Svejer)-exterior lateral view of left valve.

Figure 6 Candona (Camptocypria) balcanica (Zalanyi)-exterior lateral view of left juvenile valve.

Figure $7 \quad$ Candona (Caspiolla) liventalina (Evlachova)-exterior lateral view of left valve.

Figure $8 \quad$ Candona (Caspiolla) gracilis (Livental)-exterior later view of female right valve.

Figure 9 Candona (Caspiolla) gracilis (Livental)—exterior later view of male right valve.

(see p. 1026)

\section{PLATE 2}

Figure 1 Candona (Caspiolla) gracilis (Livental)-interior lateral view of female right valve.

Figure 2 Candona $(C$.) gracilis (Livental)-interior lateral view of juvenile right valve (larval stage 6).

Figure 3 Idem-larval stage 5.

Figure 4 Candona (Caspiocypris) elongata (Svejer)—interior lateral view of left valve.

Figure 5 Hemicytherura $\mathrm{sp}_{\mathrm{1}}$-interior lateral view of right valve.

Figure 6 Candona (Caspiolla) ex gr. flectimarginata Sokac-interior lateral view of right valve.

Figure 7 Candona (Caspiolla) liventalina (Evlachova)-interior lateral view of left valve.

Figure $8 \quad$ Candona $\mathrm{sp}_{3}$ ex gr. nonreticulata Sokac-interior lateral view of left valve.

(see p. 1027) 
R. OLTEANU

PLATE 1
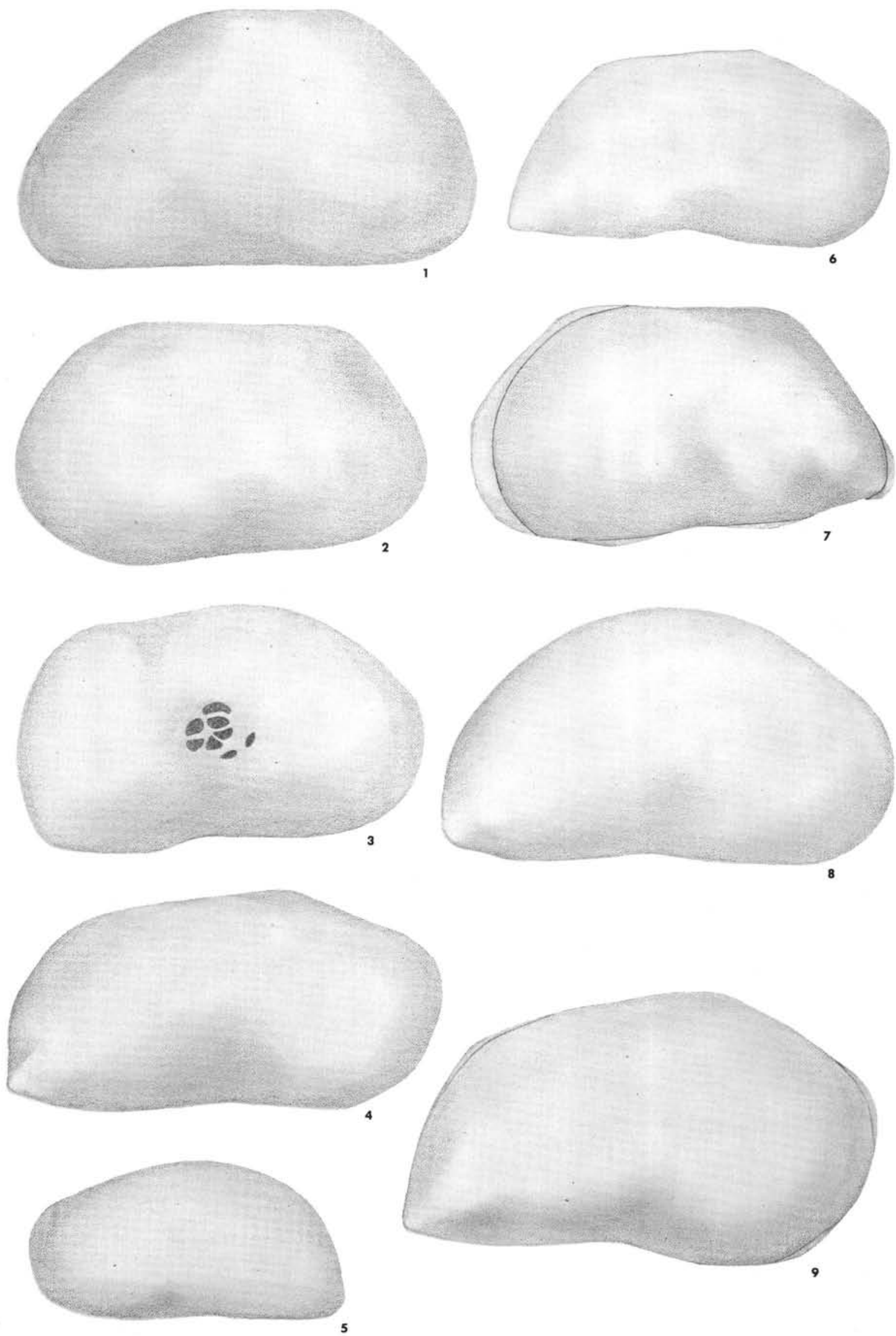
PLATE 2
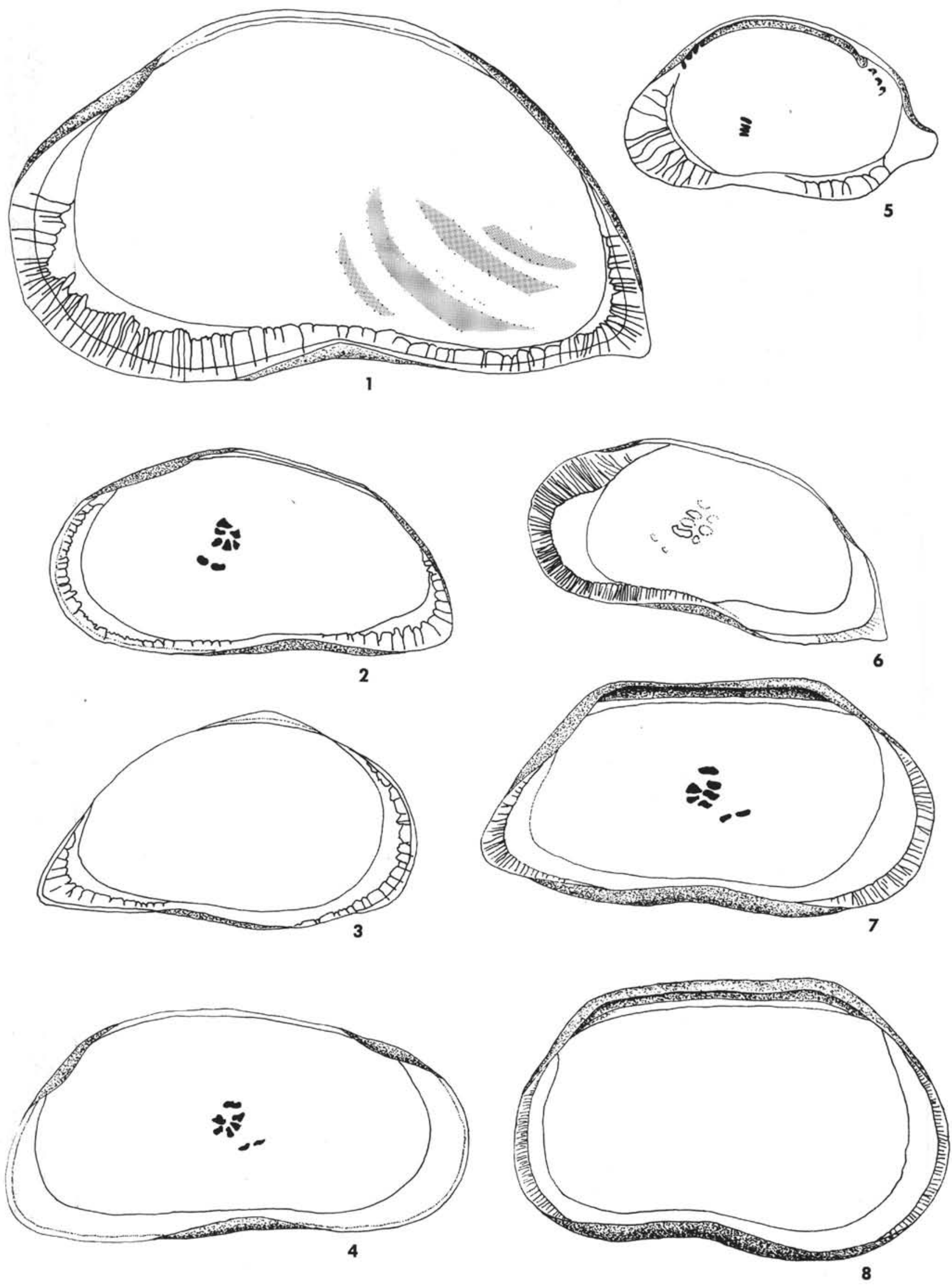


\section{PLATE 3}

Figure $1 \quad$ Paracandona aff. verrucosa (Stanceva)-interior lateral view of right valve.

Figure 2 Paracandona aff. verrucosa (Stanceva)-dorsal view.

Figures 3, 4, 6 Candona (Pontoniella) acuminata striata Mandelstam-exterior lateral view of different morphotype.

Figures 5,7 Candona (Pontoniella) acuminata striata Mandelstam-interior lateral view of different morphotype.

\section{PLATE 4}

Figure $1 \quad$ Leptocythere ergeniensis (Svejer)-exterior lateral view of left valve.

Figure 2 Leptocythere bosqueti (Livental)—exterior lateral view of left valve.

Figure 3 Leptocythere aff. nostrata (Livental)-exterior lateral view of left valve.

Figure 4 Leptocythere aff. caspia (Livental)-exterior lateral view of left valve.

Figure $5 \quad$ Leptocythere adrusovi (Livental)-interior lateral view of right valve.

Figure 6 Leptocythere bosqueti (Livental)-interior lateral view of left valve.

Figure $7 \quad$ Leptocythere striatocostata (Svejer)-exterior lateral view of right valve.

Figure $8 \quad$ Leptocythere aff. cymbula (Livental)-exterior lateral view of right valve. 
PLATE 3
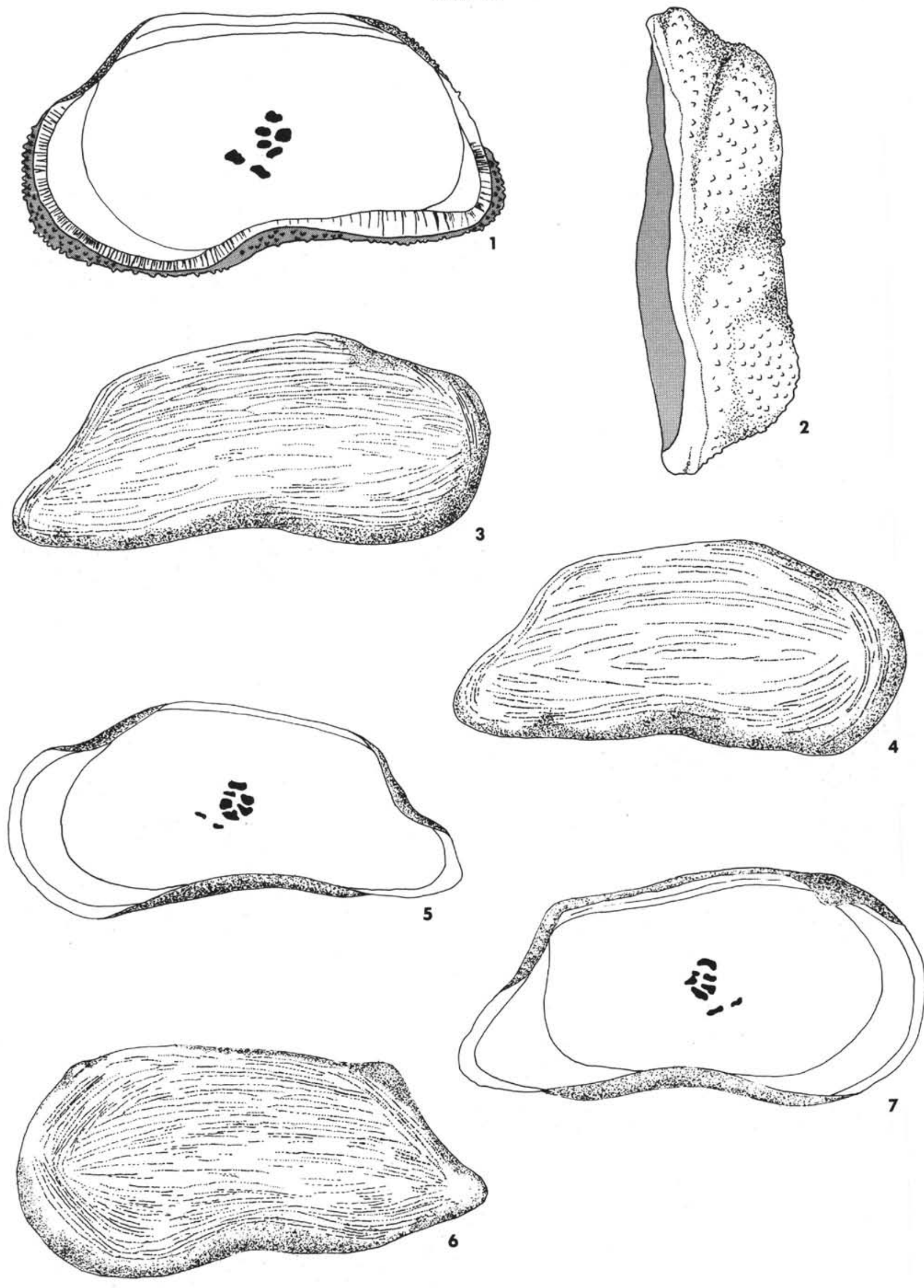

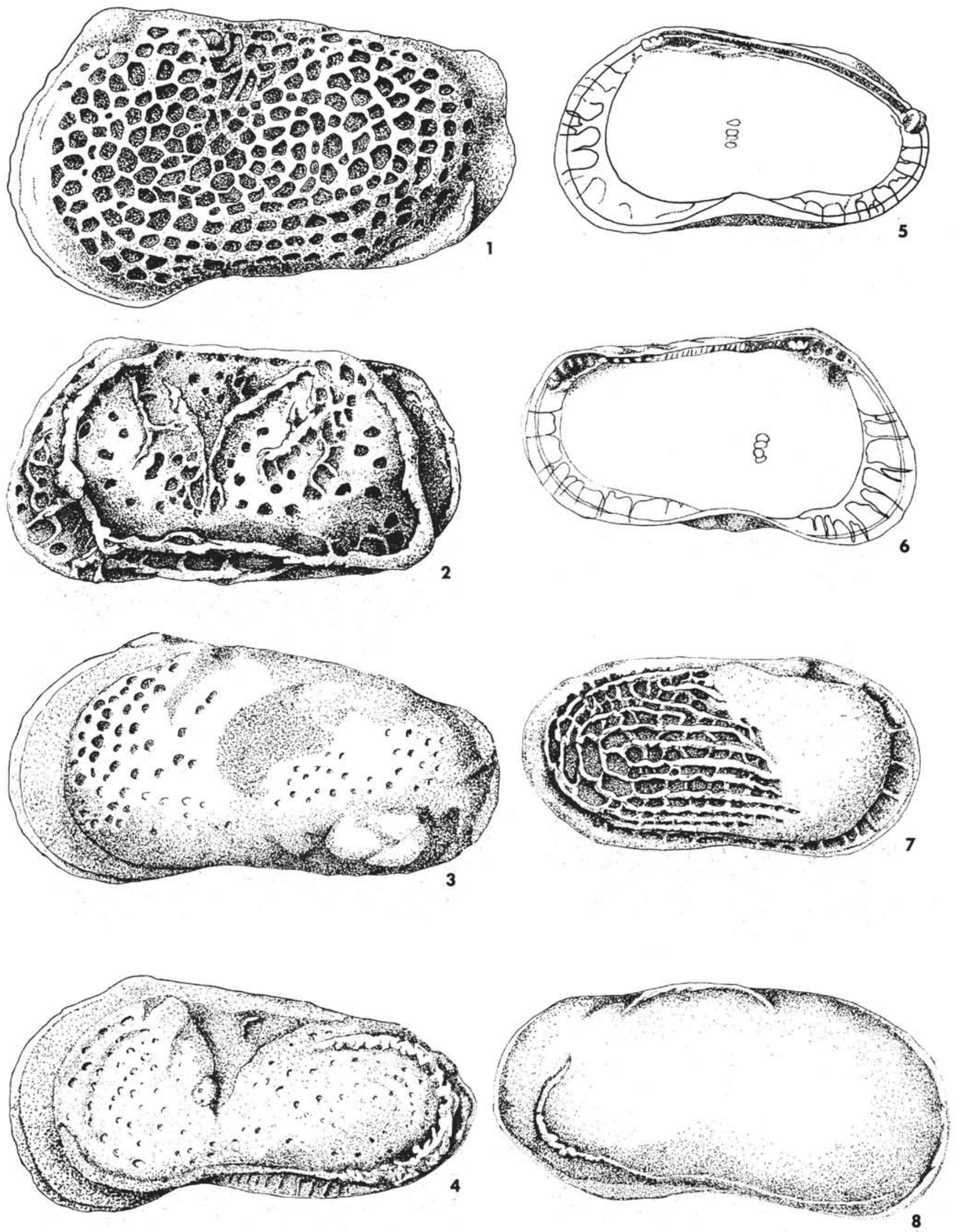


\section{PLATE 5}

Figure $1 \quad$ Leptocythere bacuana (Livental)—exterior lateral view of juvenile left valve.

Figure 2 Leptocythere aff. cymbula (Livental)-interior lateral view of right valve.

Figure 3 Leptocythere ergeniensis (Svejer)—interior lateral view of right valve.

Figure $4 \quad$ Callistocythere cf. diffusa (G.W. Müller)-interior lateral view of left valve.

Figure $5 \quad$ Cytherissa bogatschovi plana Klein-interior lateral view of right valve.

Figure $6 \quad$ Loxoconcha eichwaldi Livental-interior lateral view of right valve.

Figure 7

Idem-interior lateral view of juvenile right valve.

Figure $8 \quad$ Loxoconcha petasa Livental - interior lateral view of left valve.

(see p. 1032)

\section{PLATE 6}

Figure 1

Cytherissa bogatschovi plana Klein-exterior lateral view of right valve.

Figure 2 Candona (Caspiolla) ex gr. flectimarginata Sokac-exterior lateral view of right valve.

Figure 3 Bacunella dorsoarcuata (Zalanyi)-exterior lateral view of left valve.

Figure 4

Bacunella dorsoarcuata (Zalanyi)—dorsal view.

Figure 5 Heterocythereis $\mathrm{sp}_{1}$-exterior lateral view of juvenile left valve.

Figure $6 \quad$ Leptocythere bosqueti (Livental)—exterior lateral view of juvenile (?) left valve.

Figure $7 \quad$ Cytherissa bogatschovi.

(see p. 1033) 


\section{PLATE 5}
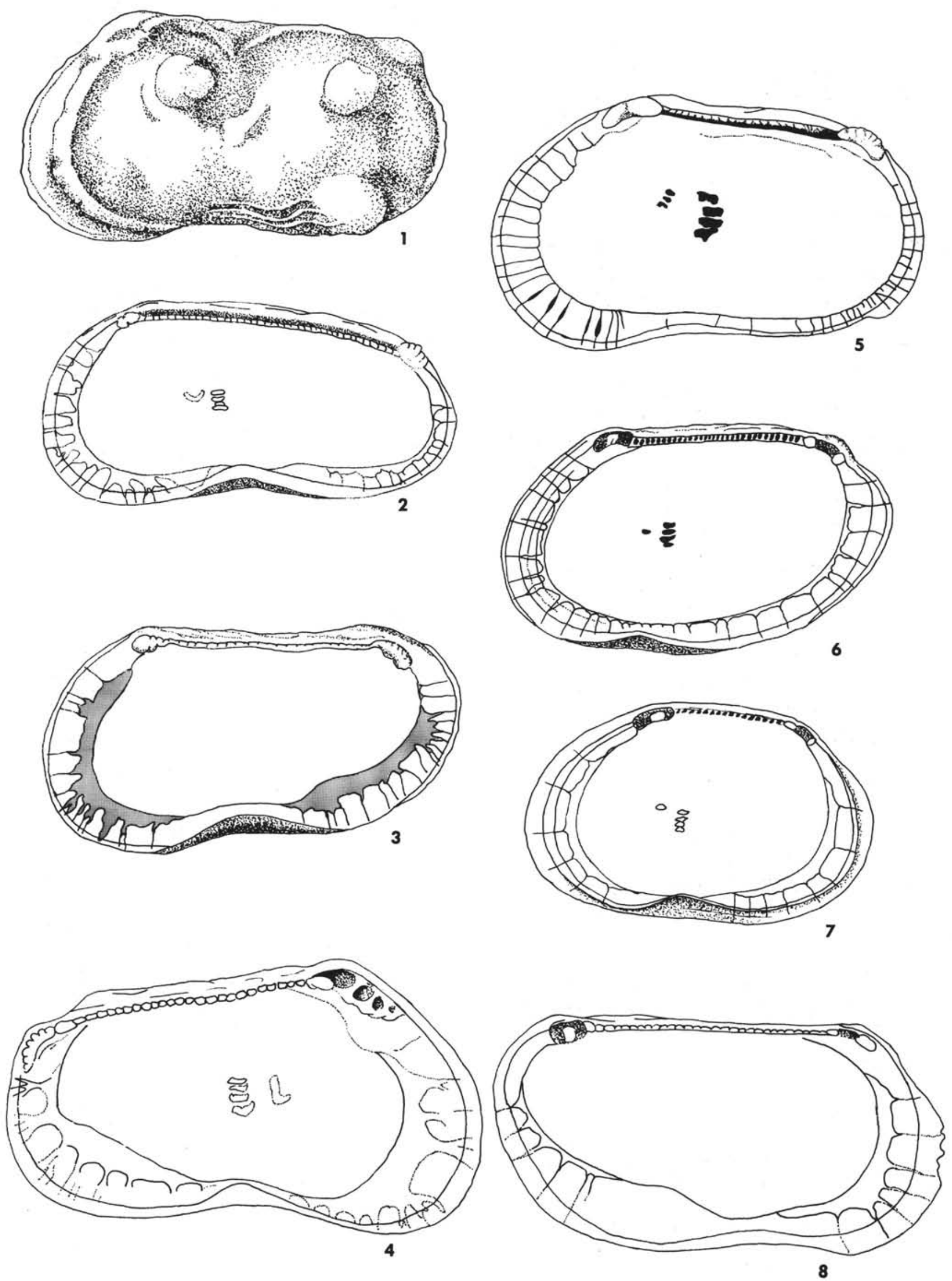
PLATE 6
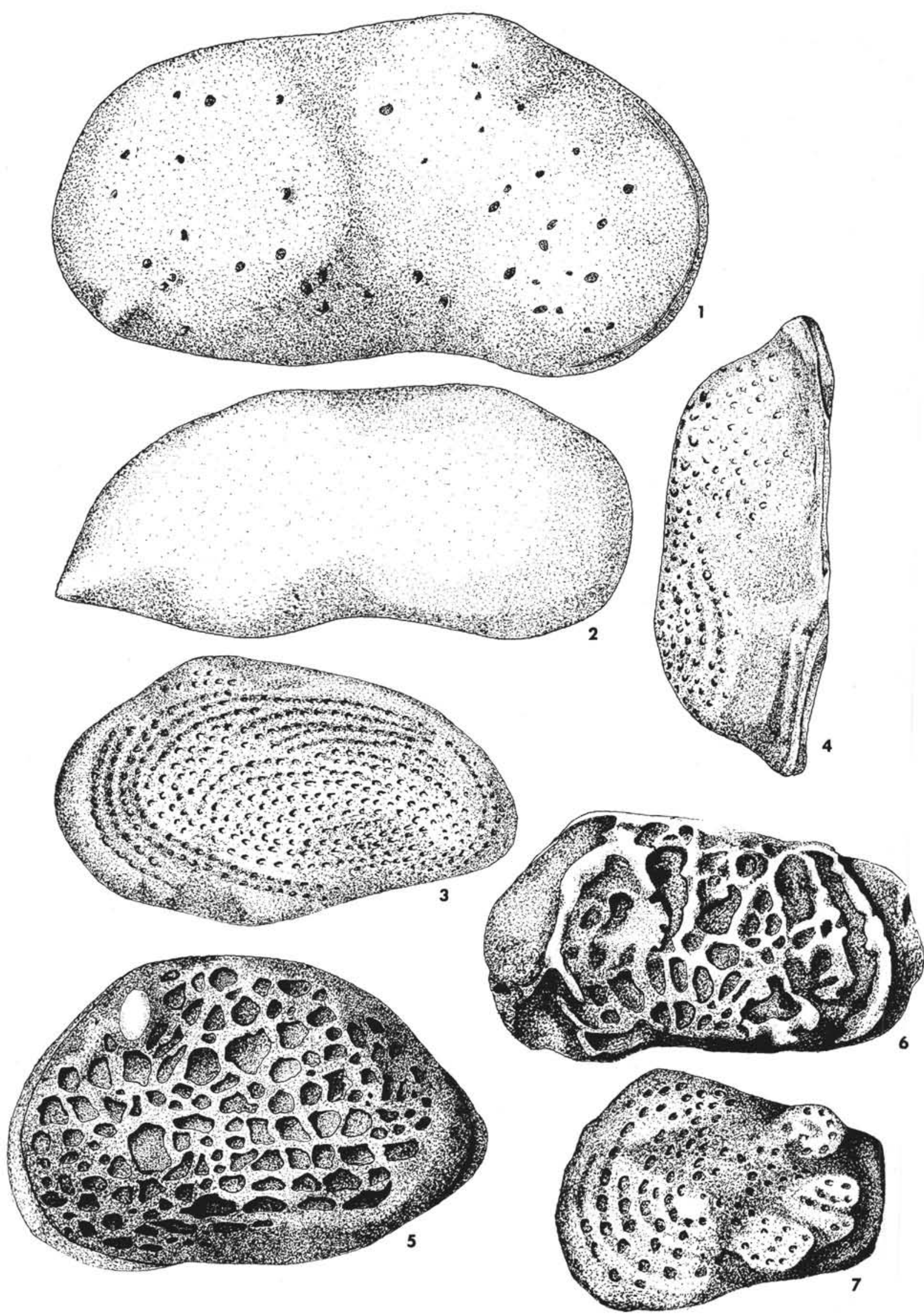


\section{PLATE 7}

Figure 1 Loxoconcha petasa Livental-exterior lateral view of left valve.

Figure 2 Loxoconcha eichwaldi Livental-exterior lateral view of right valve.

Figure 3 Leptocythere aff. cymbula (Livental)-exterior lateral view of right valve.

Figure 4 Leptocythere multituberculata (Livental)-exterior lateral view of left valve.

Figure $5 \quad$ Leptocythere andrusovi (Livental)—exterior lateral view of right valve.

Figure 6 Cytheroma (?) $\mathrm{sp}_{\mathrm{l}}$-exterior lateral view of left valve.

Figure $7 \quad$ Hemicytherura $\mathrm{sp}_{\mathrm{l}}$-exterior lateral view of right valve.

Figure $8 \quad$ Loxoconcha ornata ornata Schneider-exterior lateral view of juvenile right valve.

Figure 9 Leptocythere aff. ergeniensis (Svejer-exterior lateral view of juvenile right valve.

Figure $10 \quad$ Callistocythere cf. diffusa (G.W. Müller)-exterior lateral view of right valve.

Figure $11 \quad$ Leptocythere bosqueti (Livental)—exterior lateral view of left valve.

\section{PLATE 8}

Figure 1

Figure 2

Figure 3

Figure 4

Figure 5

Figure 6

Figure 7
Loxoconcha ornata ornata Schneider-exterior lateral view of right valve.

Idem-ventral view.

Idem-exterior lateral view of juvenile right valve.

Idem-dorsal view.

Idem-interior lateral view of juvenile right valve.

Loxoconcha schweyeri Suzin-exterior lateral view of left valve.

Paracandona aff. verrucosa (Stanceva)-exterior lateral view of right valve. 

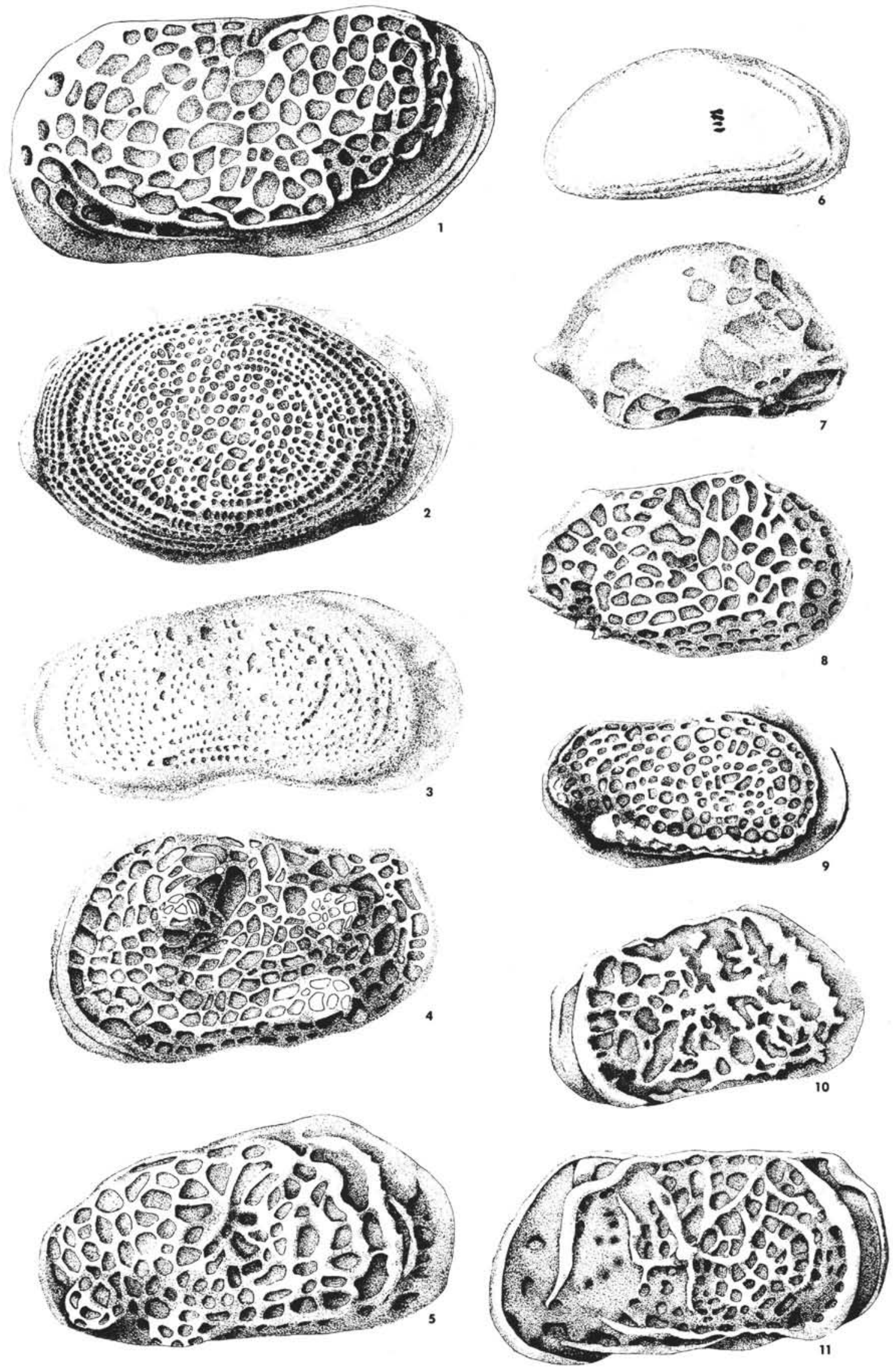


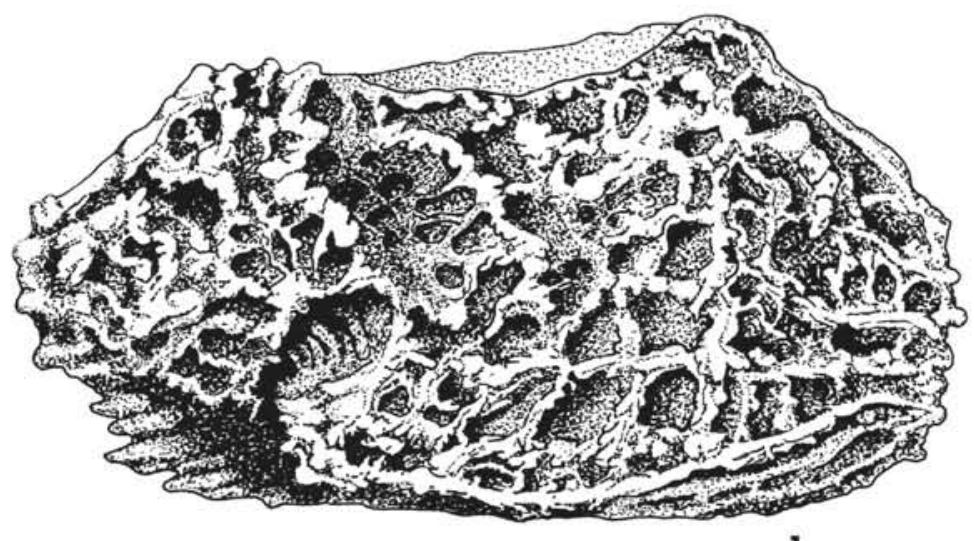

1
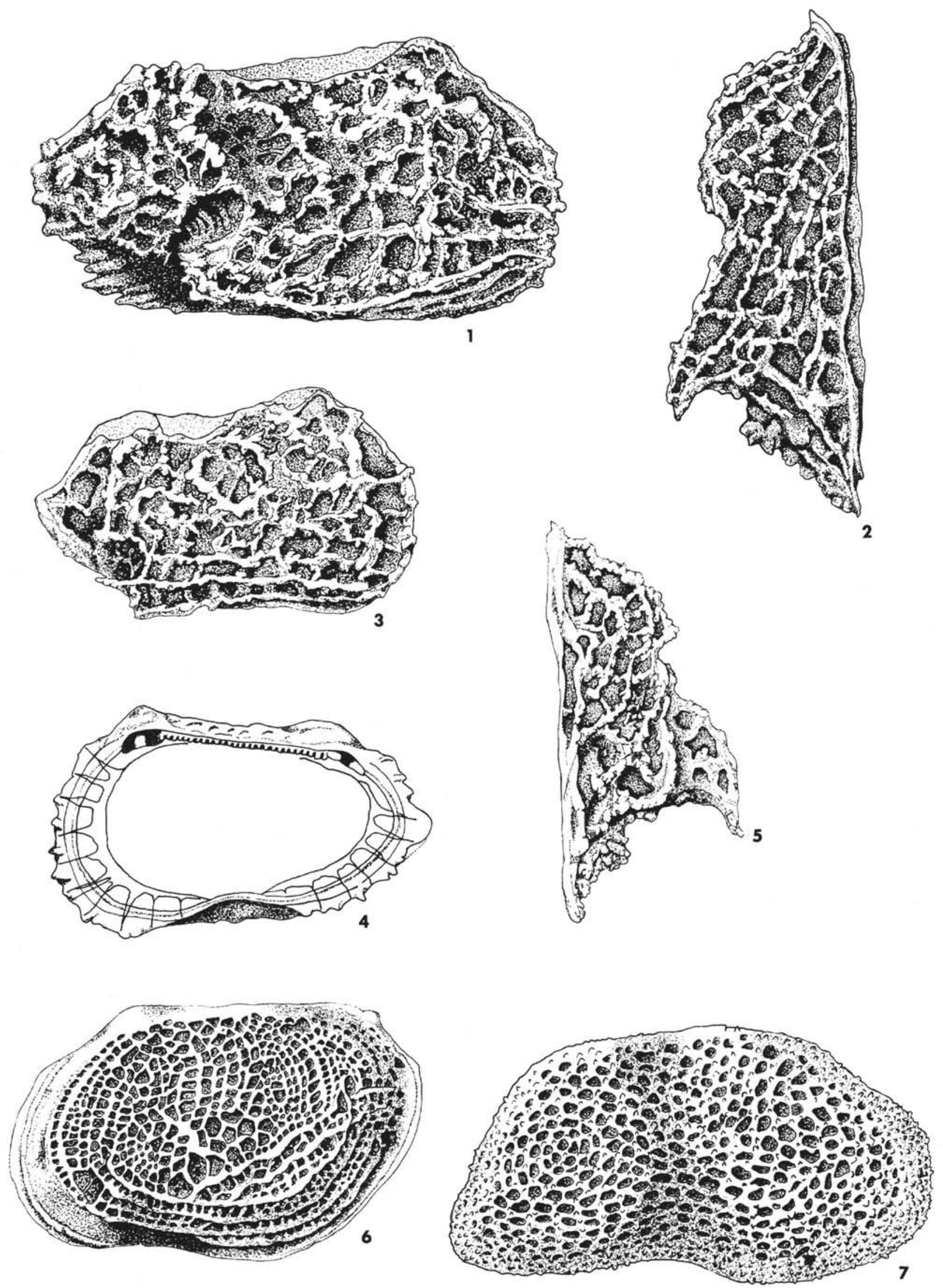


\section{PLATE 9}

Figure $1 \quad$ Candona (Caspiolla) balcanica (Zalanyi)—exterior lateral view of left valve.

Figure 2 Idem-interior lateral view of left valve.

Figure 3 Amplocypris aff. subacuta Zalanyi-exterior lateral view of left valve.

Figure 4 Idem-interior lateral view of left valve.

Figure 5 Candona (Pontoniella) schemachensis Mandelstam-exterior lateral view of left valve.

Figure 6 Candona (Pontoniella) $\mathrm{sp}_{1}$-exterior lateral view of right valve.

Figure 7 Candona (Caspiolla) confragosa Markova-exterior lateral view of left valve.

Figure $8 \quad$ Leptocythere bacuana (Livental)—exterior lateral view of left valve.

(see p. 1038) 
PLATE 9
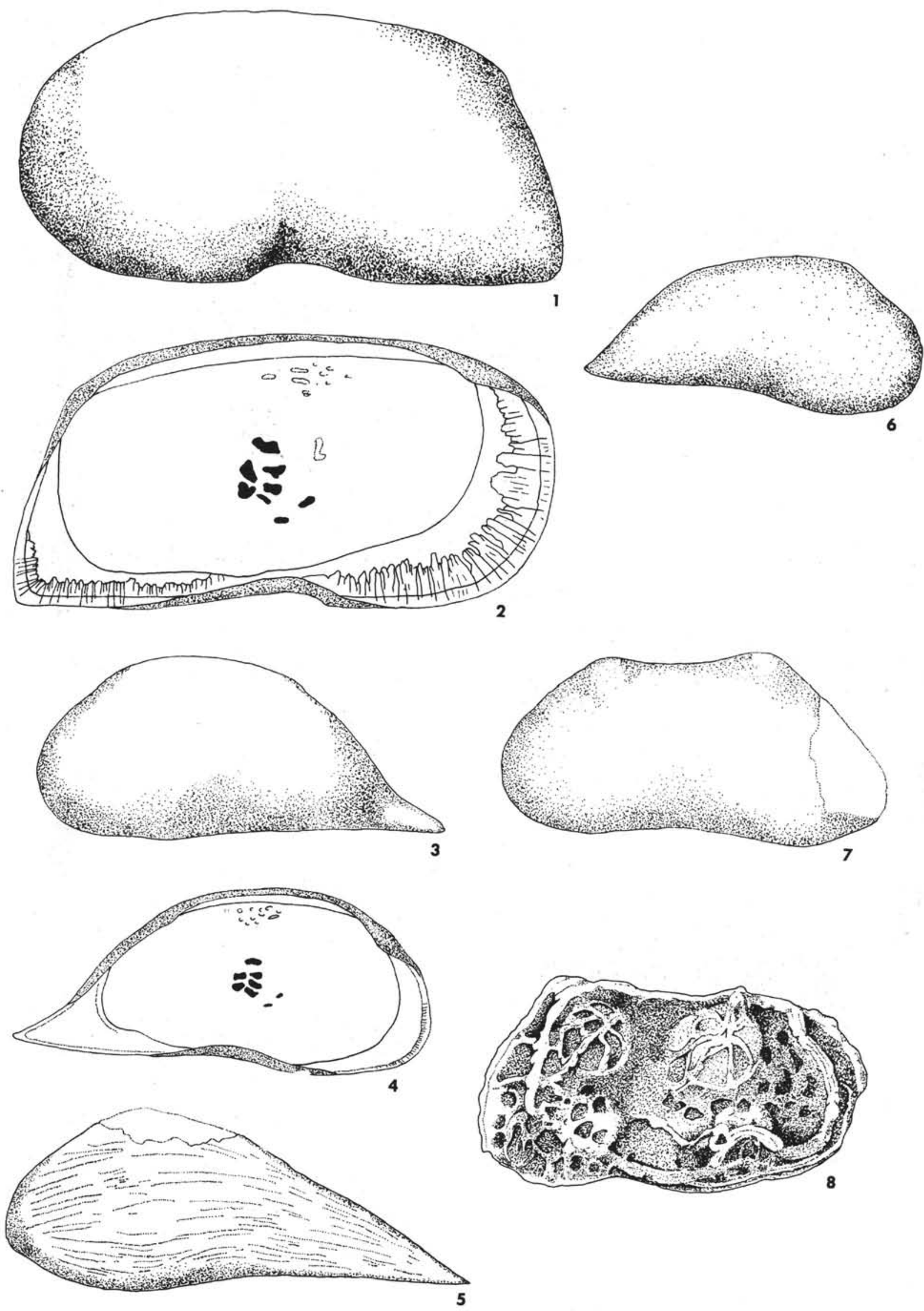